\title{
Hydralazine Reverses Stress-Induced Elevations in Blood Pressure, Angiotensin II, Testosterone, and Coronary Pathology in a Social Colony Model
}

\author{
Changying Li, Ron Salisbury, and Daniel Ely \\ Department of Biology, University of Akron, Akron, OH 44325-3908, USA \\ Correspondence should be addressed to Daniel Ely, ely1@uakron.edu \\ Received 4 April 2011; Accepted 18 May 2011 \\ Academic Editor: A. Wincewicz
}

Copyright $\odot 2011$ Changying Li et al. This is an open access article distributed under the Creative Commons Attribution License, which permits unrestricted use, distribution, and reproduction in any medium, provided the original work is properly cited.

\begin{abstract}
Background. The vasodilator hydralazine (HYZ) has been used successfully to lower blood pressure (BP) in hypertension. Recently proposed novel mechanisms suggest it may be an important drug in reducing cardiovascular pathology. Methods. The hypothesis was that social stress would increase BP and endocrine factors that lead to increased coronary artery collagen deposition, and that HYZ treatment would reverse these changes. SHR males were compared: controls, colony stress and colony stress with HYZ treatment. Results. BP was significantly elevated in the stress group compared to controls and HYZ reduced the BP compared to controls. Plasma Ang II and T were significantly increased by colony stress compared to controls and HYZ restored both to control values. Collagen deposition in the coronary artery was increased in the colony stress group compared to controls but HYZ treatment restored the collagen to that of control values. Conclusions. HYZ reduced not only BP, but also reduced coronary adventitial collagen. The mechanism of the BP effect is most likely through vasodilation and the collagen reduction may be due to both a direct action of HYZ on collagen synthetic enzymes and indirectly through an effect of testosterone and angiotensin II.
\end{abstract}

\section{Introduction}

Hypertension is a major risk factor for coronary heart disease, and a common cause of stroke, heart failure, and kidney failure. Hypertension is associated with increased peripheral vascular resistance, cardiac output, and decreased blood flow to tissues [1]. It increases shear force that injures the endothelial wall of blood vessels and increases deposition of collagen in myocardium and blood vessels [2]. A widely used animal model of hypertension is the spontaneously hypertensive rat (SHR). Mechanistic studies suggest that the sympathetic nervous system (SNS) is involved in this model of hypertension [3] and chronic elevation of SNS activity leads to increased blood pressure (BP) and organ pathology [4]. Various forms of acute and chronic stress lead to activation of the SNS, elevated BP and renal and cardiovascular injury [5-8]. Various types of pharmacological treatment successful lower BP but including hydralazine (HYZ), but novel HYZ mechanisms suggest new ways that coronary vessel pathology may be altered.
In the following study, we examined the effects of chronic social stress on plasma testosterone ( $\mathrm{T}$ ), angiotensin II (Ang $\mathrm{II})$, norepinephrine (NE), and BP on morphologic changes in the coronary arteries of male SHR rats. We hypothesized that social stress would increase BP and endocrine factors that lead to increased coronary pathology characterized by collagen deposition and that by lowering the BP with HYZ the endocrine changes, BP and consequent coronary pathology would be reduced to control levels.

\section{Methods}

2.1. Animals. The hypertensive SHR strain was acquired from Harlan Sprague Dawley Inc. (Indianapolis, Ind, USA) who obtained the original breeding stocks from the National Institute of Health (NIH). The colony has been bred and maintained in the Biology Resource Center (BRC) at the University of Akron since 1981. All protocols were approved by the IACUC and followed NIH and APS guidelines for animal welfare. Breeding cages were set up for adult SHR rats 
with one male and three females per cage $(51 \times 41 \times 22 \mathrm{~cm})$. Pups were nursed by their mother and weaned and separated by sex at four weeks of age. Three experimental groups of SHR (6 weeks of age) were used: a control group, a colony stress group and a colony stress group with HYZ treatment. The experimental duration was 8 weeks (6-14 weeks of age). Standard cages $(48 \times 27 \times 20 \mathrm{~cm})$ containing two males each were used for the control group $(n=10)$. The colony-stress group consisted of 10 male and 10 female rats that were placed in an open field box $(1.2 \times 1.2 \times 0.3 \mathrm{~m})$. On each side of the box two standard rat cages were connected via plastic tubes that permitted direct entry of animals into the large central open field area [9]. Another group was set up as a colony stress and HYZ treatment group with 10 male and 10 female rats. HYZ was administered (Sigma Chemical Co., St. Louis, Mo, USA) ( $80 \mathrm{mg} / \mathrm{L})$ in the drinking water and all groups were fed standard rat chow (Agway Inc. Country Foods Division, Syracuse, NY, USA). Room temperature of $25^{\circ} \mathrm{C}-27^{\circ} \mathrm{C}$ and a humidity of $40-50 \%$ were maintained. All animals were kept on a 12 hour light/dark cycle (6 AM to $6 \mathrm{PM}$, light/6 PM to $6 \mathrm{AM}$, dark).

Males in the colony-stress group and the colony-stress HYZ group were exposed to the social stresses of competing for territory, food and water, and females. In addition, social stress was compounded by rotating the outside cages of the open field box clockwise every other day without changing the bedding. By using this method, the territories of the rats continually changed thereby increasing colony stress levels $[5]$.

2.2. Systolic Blood Pressure Measurement. Systolic blood pressure (SBP) was taken by the tail cuff sphygmomanometry every other week from week 6 to 14 [10]. Each rat was placed in a preheated chamber $\left(40^{\circ} \mathrm{C}\right)$ for about 10 to 15 minutes. The size of the tail cuff used varied with the age of the rats. A total of five SBP measurements were taken for each rat and averaged to give an overall BP per rat. Each measurement was taken on the same day and at the same time (1000-1400 hrs).

2.3. Blood Collection. Blood was collected (13 weeks of age) for plasma norepinephrine (NE), testosterone (T), and angiotensin II (Ang II) analysis. Each rat was anaesthetized with 3\% sodium Brevital (Eli Lilly, Indianapolis, IN, $75 \mathrm{mg} / \mathrm{Kg}$, i.p.) and bled by retro-orbital puncture [11]. The samples were centrifuged at $4^{\circ} \mathrm{C}$ for ten minutes at $5000 \times \mathrm{g}$ 's and plasma was stored at $-70^{\circ} \mathrm{C}$.

2.4. Hormone Measures. HPLC with electrochemical detection was used to measure plasma NE and adrenal gland NE [12]. Adrenal glands were homogenized in ice cold mobile phase, protein removed, and NE assayed by HPLC [13]. T was analyzed using a coated tube radioimmunoassay (RIA) kit (Bio Rad Laboratories, Hercules, Calif, USA). Tri-level controls were run to verify accuracy. Plasma Ang II levels were measured by Angiotensin II I ${ }^{125}$ RIA kit (American Laboratory Products company, Ltd. P.O. Box 451, Windham, $\mathrm{NH})$.

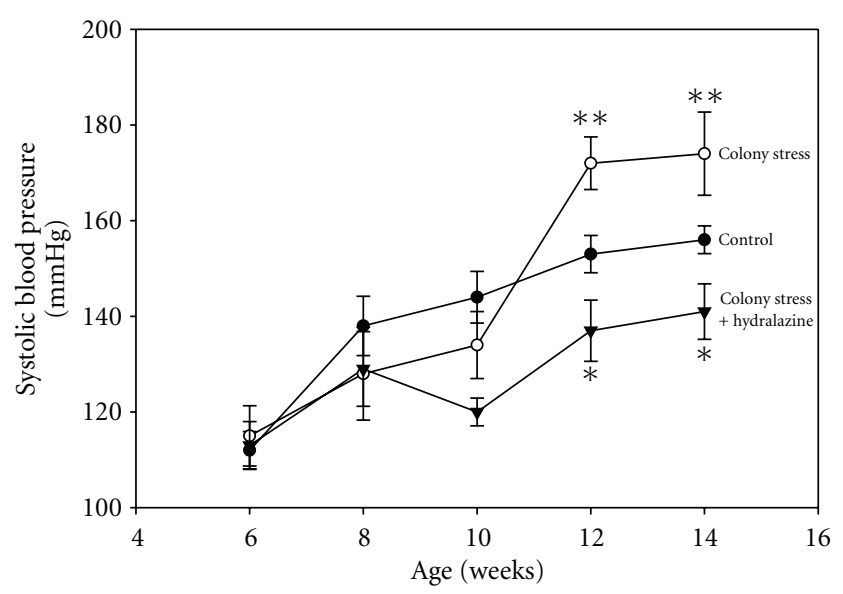

FIGURE 1: Systolic blood pressure by group and time (open circles = colony stress group, solid circles $=$ control group, solid triangles = colony stress and hydralazine group, means, \pm s.e.m., two way repeated measures ANOVA: treatment $F=3.57, d f=2, P<0.05$, time: $F=33.3, d f=4, P<0.001$ and interaction: $F=2.47$, $d f=8, P<0.05)$. Following the two-way ANOVA, $t$-tests showed that the colony stress group had elevated BP at ages 12 and 14 weeks compared to controls $\left({ }^{* *} P<0.01\right)$ and hydralazine reduced the BP at 12 and 14 weeks compared to controls $(* P<0.05)$.

2.5. Histologic Analysis. At fifteen weeks of age the rats were terminated, and the hearts and adrenal glands removed, weighed, and the adrenals stored at $-70^{\circ} \mathrm{C}$ for $\mathrm{NE}$ analysis. The left ventricle was fixed in 10\% neutral buffered formalin and embedded in paraffin. The sections were sliced at $6 \mathrm{u}$ and mounted on slides coated with 3-aminopropyl-trithoxysilane and stained with sirius red to measure collagen deposition in coronary vessel walls $[5,6]$. Micrographs were taken of representative sections from each group (Olympus BX60F-3, Melville, NY, USA) using a digital camera (MTI DC330, Michigan City, Ind, USA) at a magnification of 200x. A scoring technique was used where a score of "zero" was given to a "normal" collagen appearance, a score of $+1=$ $10 \%-20 \%$ increase in adventitial collagen, $+2=20 \%-30 \%$ increase, $+3=30 \%-40 \%$ increase, and a $+4=40 \%-50 \%$ increase [6]. This scoring method was based on a previous scoring system used for rat [14] and mouse tissues [15]. Tissues were scored by three individuals who were blind to the treatment group and the final score assigned was the average of the three scores.

2.6. Statistics. A two-way ANOVA with repeated measures for BP (time and treatment variables) was performed followed by Bonferonni's pairwise comparison. For the hormone variables and coronary scoring, Students $t$-tests were performed. The significance was set at $P<0.05$.

\section{Results}

3.1. Systolic Blood Pressure. BP reached hypertensive levels in both the control and stress groups at 12 and 14 weeks of age and HYZ treatment returned the BP to control levels (Figure 1 two-way repeated measures ANOVA: treatment: 


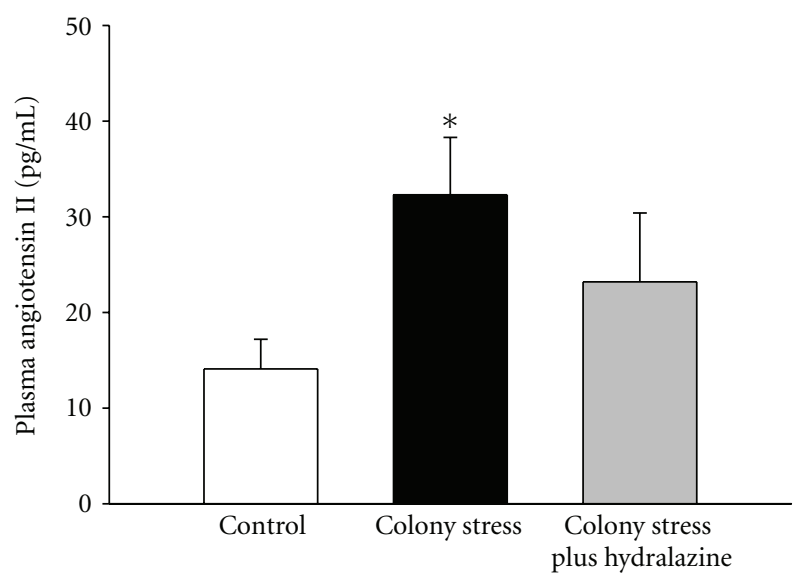

(a)

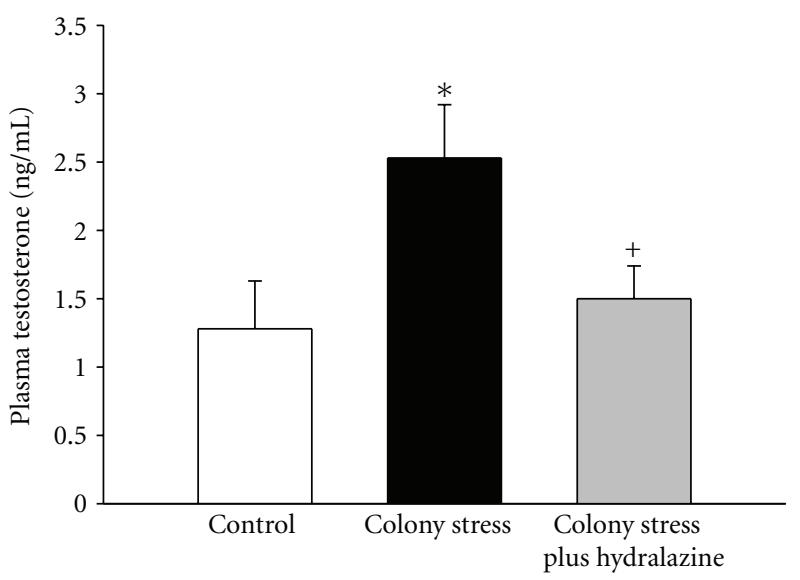

(b)

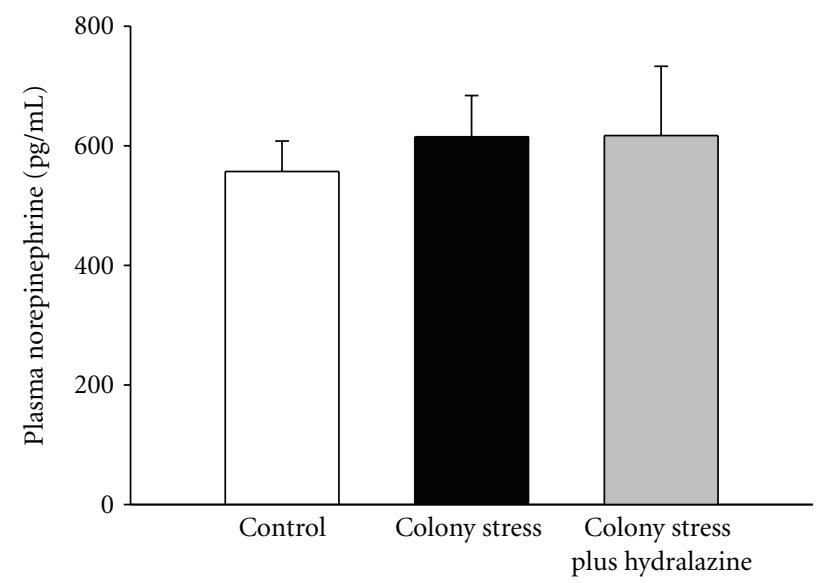

(c)

Figure 2: Plasma angiotensin II (a), plasma testosterone (b), and plasma norepinephrine (c) at 13 weeks of age by group (means, \pm s.e.m., ${ }^{*} P<0.05$ compared to controls, ${ }^{+} P<0.05$ compared to colony stress).

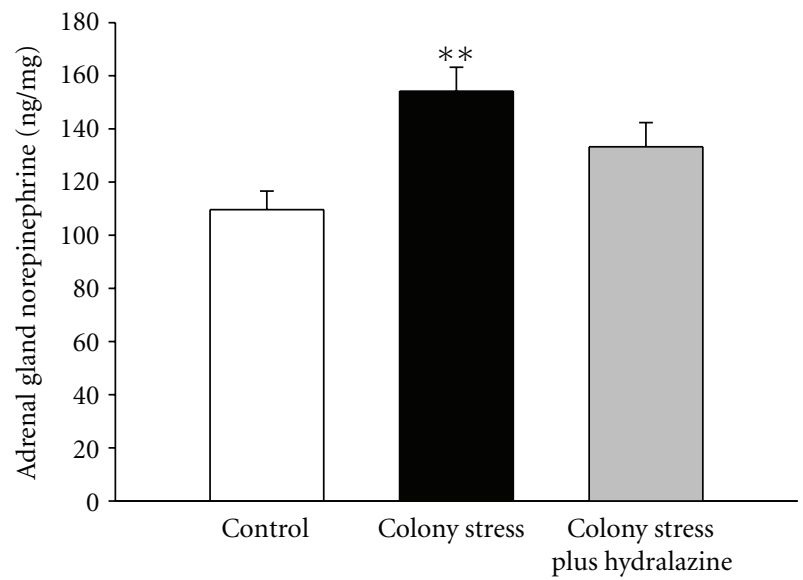

FIGURE 3: Adrenal gland norepinephrine content at 13 weeks by group (means, \pm s.e.m., ${ }^{* *} P<0.01$ ).

$F=3.57, d f=2, P<0.05$, time: $F=33.3, d f=4, P<0.001$ and interaction: $F=2.47, d f=8, P<0.05)$.

3.2. Hormone Levels. Figure 2 shows hormone levels at 13 weeks of age. Plasma Ang II and $\mathrm{T}$ were significantly increased by colony stress compared to controls $(P<0.05)$ and HYZ treatment restored both to control values (Figures 2(a) and 2(b)). There were no significant differences in plasma NE levels between groups (Figure 2(c)). However, adrenal gland NE content was elevated in the colony stress group compared to controls $(P<0.01)$ and HYZ restored the NE levels to that of controls (Figure 3).

3.3. Histology. Sirius red stained the coronary adventitial collagen a red color, and collagen deposition was highest in the colony stress group compared to controls $(P<$ 0.001), but HYZ treatment restored the collagen to that of control values (micrographs Figure 4). Figure 5 shows the quantification of this data.

\section{Discussion}

Our laboratory and others have shown that the social stress of territorial defense, competition for females, food, and water combined with box rotation raises $\mathrm{BP}$ and is accompanied by renal and heart pathology $[5,16,17]$. Even in normotensive WKY rats, chronic social stress of colony interaction caused a significant rise in BP and coronary collagen deposition [5]. 

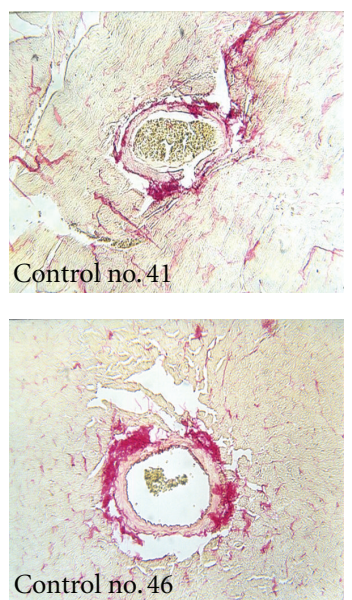

(a) Controls
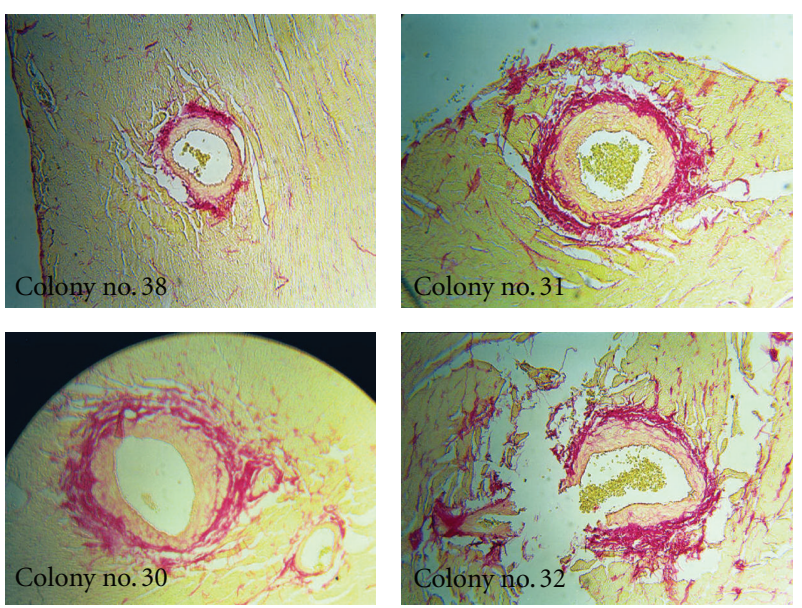

(b) Colony stress
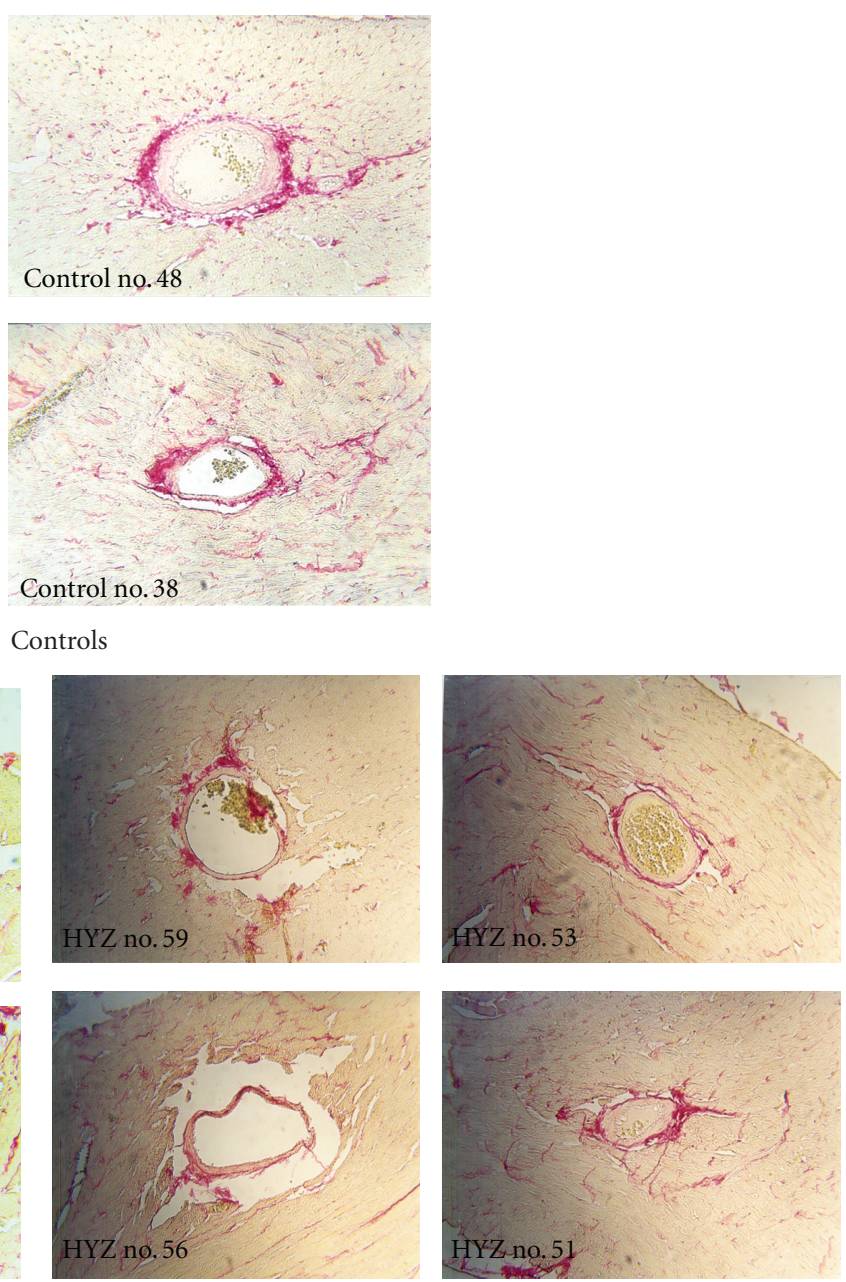

(c) Colony stress plus hydralazine

FIGURE 4: Representative micrographs of coronary artery by group $((a)=$ controls, $(b)=$ colony stress, $(c)=$ colony stress plus hydralazine, magnification $=200 \mathrm{x}$, red staining $=$ adventitial collagen $)$.

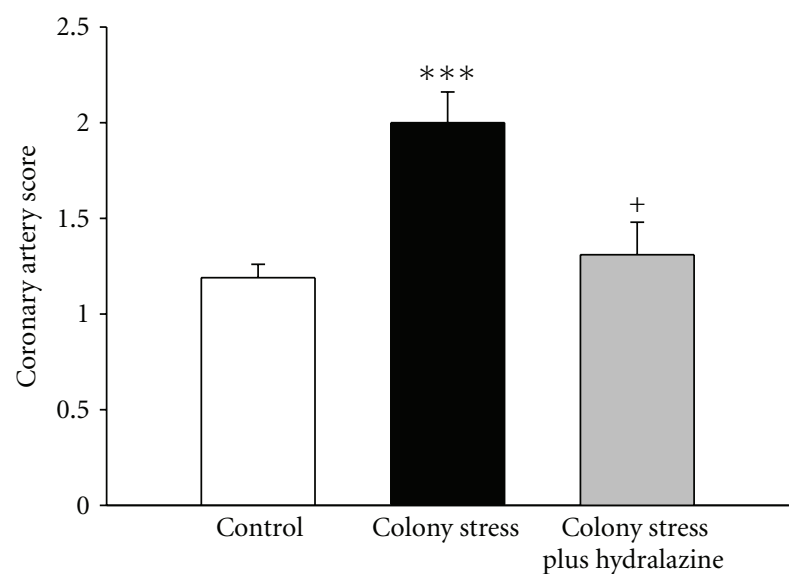

FIGURE 5: Coronary artery score by group (means, \pm s.e.m., ${ }^{* * *} P<$ 0.001 compared to control, ${ }^{+} P<0.05$ compared to colony stress).

HYZ is a vasodilator but is usually not a primary drug to treat hypertension, because it can elevate SNS activity, cause salt retention and elevate plasma renin activity [18]. In the SHR, 5 weeks of HYZ treatment increased left ventricular NE turnover $25 \%$ probably as a compensation to the lowered BP, however there was no change in plasma NE [19]. Also, HYZ can increase plasma renin [20]. For instance, in the two kidney-1 clip rat hypertensive model, HYZ lowered BP, but even after 56 days of treatment, plasma renin activity was elevated $100 \%$ [21]. HYZ is thought to reduce peripheral resistance directly by relaxing the smooth muscle cell layer in arterial vessels, but other mechanisms also function, such as altered calcium balance in vascular smooth muscle cells that inhibits calcium release from the sarcoplasmic reticulum [22]. This prevents contraction mediated by calcium-dependent ATPases, kinases [23], or ion channels. Indeed, HYZ interferes with calcium influx and release from the rat tail artery [24].

Another potential effect of HYZ is on collagen deposition in the heart and blood vessels. In SHR, chronic HYZ treatment for 5-26 months reduced BP and prevented signs of heart failure and reduced left ventricular collagen types I and III [25]. However, others have reported that HYZ did not correct the altered collagen phenotype in SHR after 26 weeks of treatment in spite of BP reduction [26]. This discrepancy may be due to timing issues and 
reversal of collagen deposition by other factors, such as, the effect of HYZ on the enzyme, collagen prolyl hydroxylase which is implicated in vascular degradation and remodeling associated with angiogenesis [27]. Indeed, HYZ treatment for 5 weeks reduced cardiac collagen in mice deficient in guanylyl cyclase-A [28].

Potential endocrine mediators for the stress induced rise in BP include chronic elevation in plasma T, Ang II, and NE, all of which are vasoconstrictors. For instance, clinical and animal studies have shown that prolonged SNS stimulation results in hypertension $[9,29]$ and neonatal chemical sympathectomy decreased BP and coronary pathology in hypertensive rats [4]. In our study, plasma NE was not significantly different at 13 weeks perhaps due to adaptation to the colony stress. This finding is supported by another study in which NE was elevated initially with colony stress but returned to normal with time. Similar results were found in a study using Sprague-Dawley normotensive rats that adapted to chronic stress after eight weeks and exhibited normal urinary norepinephrine and epinephrine levels [30]. In yet another study using SHR, 5 weeks of HYZ treatment increased left ventricular NE turnover $25 \%$, probably as a compensation to the lowered BP; however, there was no change in plasma NE [19]. This is not to say that the catecholamines are not involved in the elevated BP and pathological events, since there is a large volume of research, including our own, that suggests the SNS is an important mediator of hypertension and vascular pathology $[1,4,9,14$ 16]. Indeed, the adrenal gland NE content was elevated which suggests enhanced chronic SNS activity [13].

In addition to the direct and indirect effects of HYZ, $\mathrm{T}$ influences BP and collagen deposition. The $\mathrm{T}$ levels in the colony-stress group increased by $100 \%$ over the control group, and HYZ normalized T. The most likely explanation in the colony stress group for the elevated $\mathrm{T}$ was the presence of females which stimulates the reproductive axis in the males $[31,32]$. Further evidence for elevated $\mathrm{T}$ influencing both $\mathrm{BP}$ and collagen is that castration significantly reduced (66\%) coronary adventitial collagen but $\mathrm{T}$ replacement increased the collagen towards control values [6]. Further support for the T hypothesis in accelerating collagen deposition is provided by an elegant, but much older study, by Wexler and Greenberg [8]. They showed that in normotensive male Sprague-Dawley rats there was a significant increase in BP with elevated sex steroids in communal breeders after four breeding cycles (six months) [8]. These animals developed aortic lesions, myocardial fibrosis, hyperactive adrenal glands, and elevated heart enzymes indicating cellular damage. It was speculated that competition for the females increased the stress levels that led to myocardial infarction and, in some cases, sudden death in the males. $\mathrm{T}$ levels were not measured, but were most likely elevated due to the breeder environment (males and females) and the fact that testes weight was significantly elevated as it was in our studies [33-35]. However, in many studies, T levels decrease under stress [36-38]. For instance, in humans, anticipation of battle or training stressed individuals show a corticosterone rise and a T decrease [39, 40]. Androgens can also interact with the rennin-angiotensin system as shown by a reduction in renin-containing cells in coagulating glands of mice after castration and an increase after T injection [41].

Not only did $\mathrm{T}$ increase with stress, but also plasma Ang II increased $114 \%$ over controls and is a known stimulator for vessel collagen deposition $[42,43]$. In cultured rat vascular smooth muscle cells, the addition of Ang II increased collagen synthesis in $24 \mathrm{hr}$, and the time required to reach maximum stimulation was approximately $48 \mathrm{hr}$ [44]. Ang II induced vascular wall thickening by acting directly on smooth muscle cells and enhancing the production of extracellular matrix proteins [44]. Ang II also has a mitogenic effect in mesenteric vessels in spite of HYZ treatment [45]. Not only is the renin angiotensin system involved in vascular collagen deposition but also in hypertensive heart disease. In heart failure, the myocardium of both ventricles is usually involved, irrespective of ventricular loading conditions, which suggests that circulating factors, and not only the hemodynamic load, are responsible for myocardial collagen deposition. In various in vivo models, it has been shown that myocardial fibrosis is associated with activation of circulating or local renin-angiotensinaldosterone systems [43]. Collagen synthesis, showed a significant and dose-dependent increase after incubation with Ang II in cultured adult human cardiac fibroblasts and was significantly decreased by Ang II receptor antagonists [42].

There is also an interaction between $\mathrm{T}$ and Ang II. For instance, previously, we found that in SHR males, following castration, there was a decrease in Ang II which was restored by $\mathrm{T}$ replacement [6]. In the present study, the decreased Ang II in the HYZ treatment may be a response to elevated plasma renin which is a compensation for the reduced $\mathrm{BP}$ which in turn would elevate plasma Ang II and then by negative feedback reduce Ang II. Chen et al. [46] observed an increase in renal and hepatic angiotensinogen mRNA and plasma renin activity in castrated SHR males receiving $\mathrm{T}$ replacement. In contrast, estrogen, downregulated angiotensin converting enzyme (ACE), reduced Ang II and increased the release of the vasorelaxant Ang 1-7 [47]. Although the ACE inhibitor captopril lowered BP in male SHR, there was no subsequent decrease in circulating levels of Ang II [6]. Furthermore, blocking the androgen receptor with Flutamide, significantly attenuated the development of hypertension in female TGR(mREN2)27 rats harboring the mouse Ren-2 renin gene. It also reduced heart hypertrophy and collagen deposition and histological signs of end-organ damage in the kidney [48].

\section{Conclusion}

Colony-induced stress elevated plasma T, Ang II, coronary adventitial collagen, and BP. HYZ not only reduced BP in the SHR stress model of hypertension, but also reduced coronary adventitial collagen. The mechanism of the BP reduction is most likely through vasodilation and the collagen reduction may be due to both direct actions of HYZ on collagen synthetic enzymes as well as action of T and Ang II. 


\section{Acknowledgments}

The authors are grateful to Gail Dunphy for biochemical and histological analyses, Sarah Francis for graphics, and Fieke Bryson for animal care. This research was partially supported by grants from the National Institutes of Health (no. R01HL48072-5) and the Ohio Board of Regents to the University of Akron.

\section{References}

[1] B. Folkow, "Physiological aspects of primary hypertension," Physiological Reviews, vol. 62, no. 2, pp. 347-504, 1982.

[2] C. G. Brilla, B. Maisch, and K. T. Weber, "Renin-angiotensin system and myocardial collagen matrix remodeling in hypertensive heart disease: in vivo and in vitro studies on collagen matrix regulation," Clinical Investigator, vol. 71, no. 5, pp. S35S41, 1993.

[3] K. B. Touw, J. R. Haywood, R. A. Shaffer, and M. J. Brody, "Contribution of the sympathetic nervous system to vascular resistance in conscious young and adult spontaneously hypertensive rats," Hypertension, vol. 2, no. 4, pp. 408-418, 1980.

[4] D. Wiley, G. Dunphy, H. Daneshvar, R. Salisbury, M. Neeki, and D. Ely, "Neonatal sympathectomy reduces adult blood pressure and cardiovascular pathology in $\mathrm{Y}$ chromosome consomic rats," Blood Pressure, vol. 8, no. 5-6, pp. 300-307, 1999.

[5] E. Andrews, C. Jenkins, D. Seachrist, G. Dunphy, and D. Ely, "Social stress increases blood pressure and cardiovascular pathology in a normotensive rat model," Clinical and Experimental Hypertension, vol. 25, no. 2, pp. 85-101, 2003.

[6] D. Seachrist, G. Dunphy, H. Daneshvar, A. Caplea, A. Milsted, and D. Ely, "Testosterone increases blood pressure and cardiovascular and renal pathology in SHR," Blood Pressure, vol. 9, no. 4, Article ID 817835, pp. 227-238, 2000.

[7] J. E. Lawler, G. F. Barker, J. W. Hubbard, and R. G. Schaub, "Effects of stress on blood pressure and cardiac pathology in rats with borderline hypertension," Hypertension, vol. 3, no. 4, pp. 496-505, 1981.

[8] B. C. Wexler and B. P. Greenberg, "Pathophysiological differences between paired and communal breeding of male and female sprague-dawley rats," Circulation Research, vol. 42, no. 1, pp. 126-135, 1978.

[9] D. L. Ely, A. Caplea, G. Dunphy et al., "Spontaneously hypertensive rat $\mathrm{Y}$ chromosome increases indexes of sympathetic nervous system activity," Hypertension, vol. 29, no. 2, pp. 613$618,1997$.

[10] C. Jenkins, R. Salisbury, and D. Ely, "Castration lowers and testosterone restores blood pressure in several rat strains on high sodium diets," Clinical and Experimental Hypertension, vol. 16, no. 5, pp. 611-625, 1994.

[11] V. Riley, "Adaptation of orbital bleeding technic to rapid serial blood studies," Proceedings of the Society for Experimental Biology and Medicine, vol. 104, pp. 751-754, 1960.

[12] A. Foti, S. Kimura, V. DeQuattro, and D. Lee, "Liquidchromatographic measurement of catecholamines and metabolites in plasma and urine," Clinical Chemistry, vol. 33, no. 12, pp. 2209-2213, 1987.

[13] A. Caplea, D. Seachrist, H. Daneshvar, G. Dunphy, and D. Ely, "Noradrenergic content and turnover rate in kidney and heart shows gender and strain differences," Journal of Applied Physiology, vol. 92, no. 2, pp. 567-571, 2002.
[14] J. P. Henry, Y. Y. Liu, W. E. Nadra et al., "Psychosocial stress can induce chronic hypertension in normotensive strains of rats," Hypertension, vol. 21, no. 5, pp. 714-723, 1993.

[15] J. P. Henry, P. M. Stephens, and G. A. Santisteban, "A model of psychosocial hypertension showing reversibility and progression of cardiovascular complications," Circulation Research, vol. 36, no. 1, pp. 156-164, 1975.

[16] D. L. Ely and J. Weigand, "Stress and high sodium effects on blood pressure and brain catecholamines in spontaneously hypertensive rats," Clinical and Experimental Hypertension, vol. A5, no. 9, pp. 1559-1587, 1983.

[17] D. L. Ely and J. P. Henry, "Neuroendocrine response patterns in dominant and subordinate mice," Hormones and Behavior, vol. 10, no. 2, pp. 156-169, 1978.

[18] J. G. Gerber and A. S. Nies, "Antihypertensive agents and the drug therapy of hypertension," in The Pharmacological Basis of Therapeutics, A. G. Gilman, T. W. Rall, A. S. Nies, and P. Taylor, Eds., pp. 784-813, Pergamon Press, New York, NY, USA, 1990.

[19] J. Tsoporis and F. H. Leenen, "Effects of arterial vasodilators on cardiac hypertrophy and sympathetic activity in rats," Hypertension, vol. 11, no. 4, pp. 376-386, 1988.

[20] J. A. Mannick, A. Huvos, and W. E. Hollander, "Posthydralazine renin release in the diagnosis of renovascular hypertension," Annals of Surgery, vol. 170, no. 3, pp. 409-415, 1969.

[21] J. Tsoporis and F. H. Leenen, "Effects of hydralazine on blood pressure, pressor mechanisms, and cardiac hypertrophy in two-kidney, one-clip hypertensive rats," Canadian Journal of Physiology and Pharmacology, vol. 64, no. 12, pp. 1528-1534, 1986.

[22] D. C. Ellershaw and A. M. Gurney, "Mechanisms of hydralazine induced vasodilation in rabbit aorta and pulmonary artery," British Journal of Pharmacology, vol. 134, no. 3, pp. 621-631, 2001.

[23] M. Jacobs, "Mechanism of action of hydralazine on vascular smooth muscle," Biochemical Pharmacology, vol. 33, no. 18, pp. 2519-2919, 1984.

[24] A. B. Ebeigbe and C. P. Aloamaka, "Mechanism of hydralazine-induced relaxation of arterial smooth muscle," Cardiovascular Research, vol. 19, no. 7, pp. 400-405, 1985.

[25] O. J. Tsotetsi, A. J. Woodiwiss, M. Netjhardt, R. Brooksbank, and G. R. Norton, "Attenuation of cardiac failure, dilatation, damage, and detrimental interstitial remodeling without regression of hypertrophy in hypertensive rats," Hypertension, vol. 38, no. 4, pp. 846-851, 2001.

[26] D. Mukherjee and S. Sen, "Alteration of cardiac collagen phenotypes in hypertensive hypertrophy: the role of blood pressure," Journal of Molecular and Cellular Cardiology, vol. 25, no. 2, pp. 185-196, 1993.

[27] H. J. Knowles, Y.-M. Tian, D. R. Mole, and A. L. Harris, "Novel mechanism of action for hydralazine: induction of hypoxiainducible factor-1 $\alpha$, vascular endothelial growth factor, and angiogenesis by inhibition of prolyl hydroxylases," Circulation Research, vol. 95, no. 2, pp. 162-169, 2004.

[28] Y. Li, Y. Saito, K. Kuwahara et al., "Vasodilator therapy with hydralazine induces angiotensin AT2 receptor-mediated cardiomyocyte growth in mice lacking guanylyl cyclase-A," British Journal of Pharmacology, vol. 159, no. 5, pp. 1133-1142, 2010.

[29] S. Julius and S. Nesbitt, "Sympathetic overactivity in hypertension," American Journal of Hypertension, vol. 9, no. 11, pp. 113S-120S, 1996. 
[30] J. A. Rosecrans, N. Watzman, and J. P. Buckley, "The production of hypertension in male albino rats subjected to experimental stress," Biochemical Pharmacology, vol. 15, no. 11, pp. 1707-1718, 1966.

[31] P. Mormede, V. Lemaire, N. Castanon, J. Dulluc, M. Laval, and M. Le Moal, "Multiple neuroendocrine responses to chronic social stress: interaction between individual characteristics and situational factors," Physiology and Behavior, vol. 47, no. 6, pp. 1099-1105, 1990.

[32] N. Sachser and C. Lick, "Social experience, behavior, and stress in guinea pigs," Physiology and Behavior, vol. 50, no. 1, pp. 8390, 1991.

[33] D. L. Ely and J. P. Henry, "Effects of prolonged social deprivation on murine behavior patterns, blood pressure, and adrenal weight," Journal of Comparative and Physiological Psychology, vol. 87, no. 4, pp. 733-740, 1974.

[34] B. A. Lapin and G. M. Churkovich, "Environmental change causing the development of neuroses and corticovisceral pathology in monkeys," in Society, Stress and Disease. The Psychosocial Environment and Psychosomatic Diseases, L. Levi, Ed., vol. 1, pp. 226-280, Oxford University Press, London, UK, 1971.

[35] G. T. Taylor, J. Weiss, and R. Rupich, "Male rat behavior, endocrinology and reproductive physiology in a mixed-sex, socially stressful colony," Physiology and Behavior, vol. 39, no. 4, pp. 429-433, 1987.

[36] F. Gómez, A. Lahmame, E. R. De Kloet, and A. Armario, "Hypothalamic-pituitary-adrenal response to chronic stress in five inbred rat strains: differential responses are mainly located at the adrenocortical level," Neuroendocrinology, vol. 63, no. 4, pp. 327-337, 1996.

[37] G. D. Gray, E. R. Smith, D. A. Damassa, J. K. Ehrenkranz, and J. M. Davidson, "Neuroendocrine mechanisms mediating the suppression of circulating testosterone levels associated with chronic stress in male rats," Neuroendocrinology, vol. 25, no. 4, pp. 247-256, 1978.

[38] R. M. Sapolsky, "Adrenocortical function, social rank, and personality among wild baboons," Biological Psychiatry, vol. 28, no. 10, pp. 862-878, 1990.

[39] D. C. Cumming, M. E. Quigley, and S. S. C. Yen, "Route suppression of circulating testosterone levels by cortisol in men," The Journal of Clinical Endocrinology \& Metabolism, vol. 57, pp. 671-673, 1983.

[40] L. E. Kreuz, R. M. Rose, and J. R. Jennings, "Suppression of plasma testosterone levels and psychological stress," Archives of General Psychiatry, vol. 26, no. 5, pp. 479-482, 1972.

[41] Y. Kon, D. Endoh, K. Murakami et al., "Expression of renin in coagulating glands is regulated by testosterone," Anatomical Record, vol. 241, no. 4, pp. 451-460, 1995.

[42] R. C. Funck, A. Wilke, H. Rupp, and C. G. Brilla, "Regulation and role of myocardial collagen matrix remodeling in hypertensive heart disease," Advances in Experimental Medicine and Biology, vol. 432, pp. 35-44, 1997.

[43] K. T. Weber, "Fibrosis and hypertensive heart disease," Current Opinion in Cardiology, vol. 15, no. 4, pp. 264-272, 2000.

[44] H. Kato, H. Suzuki, S. Tajima et al., "Angiotensin II stimulates collagen synthesis in cultured vascular smooth muscle cells," Journal of Hypertension, vol. 9, no. 1, pp. 17-22, 1991.

[45] E. J. Su, D. M. Lombardi, J. Siegal, and S. M. Schwartz, "Angiotensin II induces vascular smooth muscle cell replication independent of blood pressure," Hypertension, vol. 31, no. 6, pp. 1331-1337, 1998.
[46] Y. F. Chen, A. J. Naftilan, and S. Oparil, "Androgen-dependent angiotensinogen and renin messenger RNA expression in hypertensive rats," Hypertension, vol. 19, no. 5, pp. 456-463, 1992.

[47] P. E. Gallagher, P. Li, J. R. Lenhart, M. C. Chappell, and K. B. Brosnihan, "Estrogen regulation of angiotensin-converting enzyme in RNA," Hypertension, vol. 33, no. 1, pp. 323-328, 1999.

[48] O. Baltatu, C. Cayla, R. Iliescu, D. Andreev, and M. Bader, "Abolition of end-organ damage by antiandrogen treatment in female hypertensive transgenic rats," Hypertension, vol. 41, no. 3, pp. 830-833, 2003. 


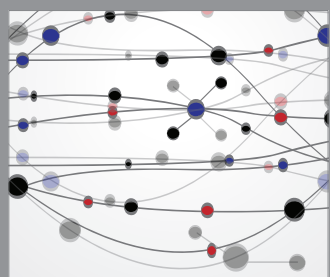

The Scientific World Journal
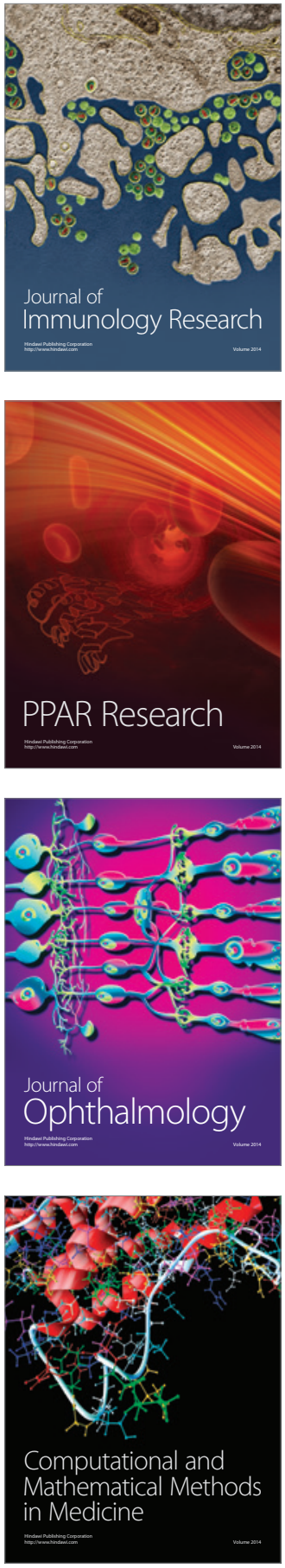

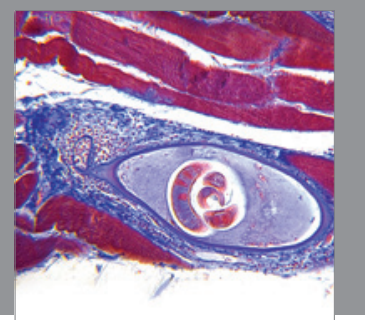

Gastroenterology

Research and Practice
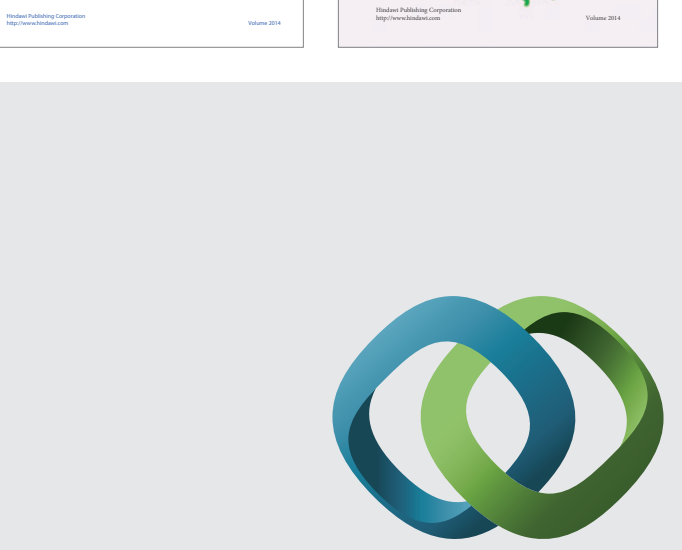

\section{Hindawi}

Submit your manuscripts at

http://www.hindawi.com
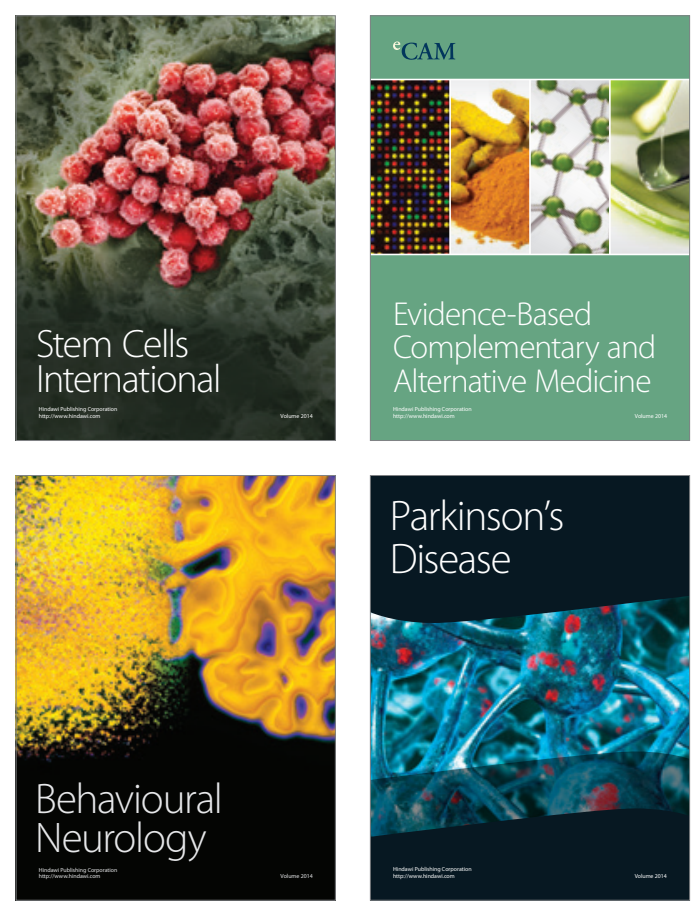

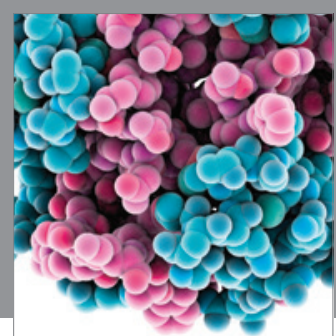

Journal of
Diabetes Research

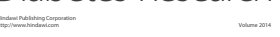

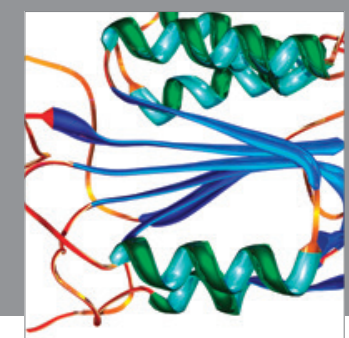

Disease Markers
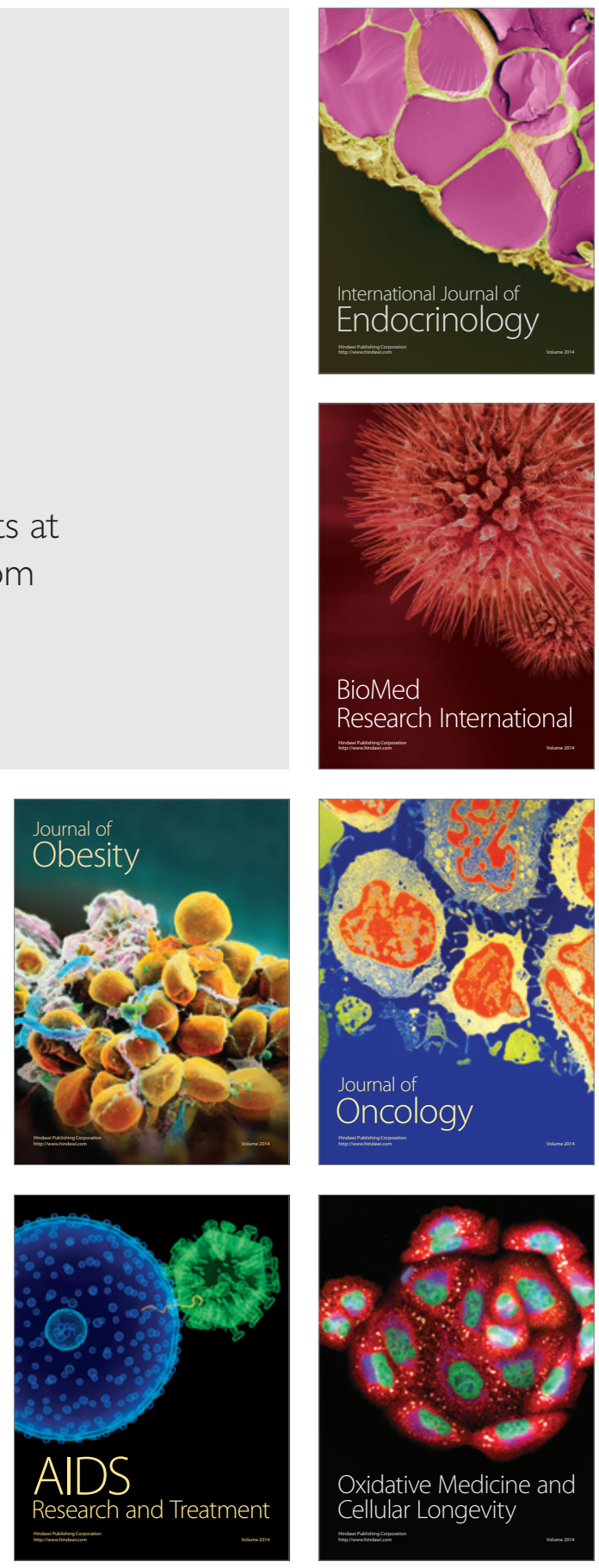